\title{
LA EDUCACIÓN EN AMÉRICA LATINA HOY EN EL HORIZONTE DE LA AGENDA EDUCATIVA POST 2015
}

Education in Latin America Today on the Horizon of the Post-2015 Education Agenda

Luis M. Lázaro Lorente*

\section{$\underline{\text { RESUMEN }}$}

El año 2030 marca el límite renovado que adopta la comunidad internacional, a través de las organizaciones gubernamentales y no gubernamentales que la representan, para el logro de los nuevos Objetivos de Desarrollo Sostenible (ODS) y de los seis objetivos del programa de Educación para Todos (EPT). Se presenta aquí una aproximación general a algunos de los aspectos que con más fuerza definen y condicionan la actual situación de la educación en América Latina en la perspectiva de la Agenda post2015. Para ello, se abordan algunas claves condicionantes del contexto como la desigualdad social y económica que caracteriza a la región. Se analizan asimismo las Declaraciones, pronunciamientos, documentos e informes generados desde el ámbito gubernamental e institucional directamente relacionados con la educación, tanto en el plano nacional como regional e internacional; también los de organizaciones de la sociedad civil comprometidas con la defensa de la educación de calidad inclusiva como derecho humano irrenunciable. De manera especial, se muestra el panorama que para América Latina presentaba en 2015 el Informe de Seguimiento de la Educación para Todos en el mundo a propósito de los

\footnotetext{
* Universitat de València (España).
} 
logros e incumplimientos regionales en sus seis Objetivos. Se concluye con una reflexión acerca de los desafíos que en educación afronta la región desde la disposición de seguir avanzando en garantizar para todos más tiempo de educación inclusiva de calidad a lo largo de la vida.

PALABRAS CLAVE: Educación en América Latina, Educación para Todos, Desigualdades en educación en América Latina, Agenda educativa post-2015.

\section{ABSTRACT}

The year 2030 marks the renewed limit adopted by the international community through its governmental and non-governmental organizations - for the achievement of the new Sustainable Development Goals (SDGs) and the six goals of the Education for All (EFA). We present here a general approach to some of the issues that define and condition the current state of education in Latin America in the wake of the post-2015 agenda. To this end, some key conditioning factors of the context, such as the social and economic inequality that characterizes the region, will be addressed. Likewise, documents generated within the governmental and institutional fields - directly related to education at all levels - as well as those coming from organizations from the civil society - committed with the defense of quality and inclusive education as a human right - will be analyzed. Special attention will be given to the picture shown by the 2015 Education for All Global Monitoring Report with regard to the achievements and failures to fulfill the six goals in Latin America. The paper ends with a reflection on the key challenges faced by the region in terms of education with the expectation of further progress in ensuring a longer period of quality and inclusive education for all throughout life.

KEY WORDS: Education in Latin America, Education for All, Inequalities in education in Latin America, Post-2015 education agenda.

$* * * * *$

\section{INTRODUCCIÓN}

Desde la celebración del Foro Mundial de Educación en Dakar en 2000, al margen de las diferencias nacionales e intranacionales, los avances globales en el ámbito de la educación en América Latina han sido significativos (POGGI, 2014: 73). En especial, en el terreno de la escolarización en los distintos niveles del sistema educativo. Las trayectorias escolares se han ampliado comenzando la escolarización más tempranamente y acabándola más tarde. Además, tomando en consideración las brechas en el acceso al sistema educativo derivadas del nivel socioeconómico de las familias de los escolares y de su área geográfica de residencia, urbana o rural, como puede observarse en la Tabla 1, se redujeron para los 
alumnos entre los 5 y los 17 años (OREALC, 2013: 22-25; SITEAL, 2015: 1-6). En buena parte, en algunos de los países, gracias a los Programas de Transferencias Condicionadas planteados en el ámbito educativo o destacado como uno de sus elementos más importantes (CECCHINI, 2014; POGGI, 2014: 60-64).

Tabla 1. Tasa de escolarización de la población por grupos de edad según nivel socioeconómico y área geográfica. América Latina, 18 países. cca. 2000, cca. 2005, cca. 2013

\begin{tabular}{|c|c|c|c|c|c|c|c|}
\hline \multirow{2}{*}{$\begin{array}{l}\text { Grupos } \\
\text { de edad }\end{array}$} & \multirow[t]{2}{*}{ Año } & \multicolumn{3}{|c|}{ Nivel socioeconómico } & \multicolumn{2}{|c|}{ Área geográfica } & \multirow[t]{2}{*}{ TOTAL } \\
\hline & & BAJO & MEDIO & ALTO & URBANA & RURAL & \\
\hline \multirow[t]{3}{*}{5 años } & 2000 & 60,4 & 81,9 & 92,4 & 80,0 & 59,8 & 74,1 \\
\hline & 2005 & 66,0 & 84,9 & 94,4 & 84,1 & 67,1 & 79,3 \\
\hline & 2013 & 71,06 & 88,3 & 95,3 & 87,7 & 72,5 & 84,3 \\
\hline \multirow{3}{*}{$\begin{array}{lrr}6 \quad \text { a } & 11 \\
\text { años } & \end{array}$} & 2000 & 91,6 & 97,5 & 99,0 & 96,4 & 91,9 & 95,1 \\
\hline & 2005 & 93,3 & 97,8 & 98,8 & 97,0 & 93,8 & 96,1 \\
\hline & 2013 & 94,9 & 98,3 & 98,5 & 97,9 & 95,3 & 97,2 \\
\hline \multirow{3}{*}{$\begin{array}{l}12 \text { a } 14 \\
\text { años }\end{array}$} & 2000 & 82,8 & 95,5 & 98,7 & 93,7 & 81,3 & 90,2 \\
\hline & 2005 & 86,3 & 96,0 & 98,4 & 94,8 & 85,8 & 92,3 \\
\hline & 2013 & 88,9 & 95,9 & 98,4 & 95,5 & 88,6 & 93,8 \\
\hline \multirow{3}{*}{$\begin{array}{lll}15 & \text { a } 17 \\
\text { años } & \end{array}$} & 2000 & 55,0 & 77,3 & 89,2 & 75,5 & 51,9 & 69,4 \\
\hline & 2005 & 59,8 & 77,9 & 88,9 & 77,7 & 57,9 & 72,5 \\
\hline & 2013 & 65,8 & 79,3 & 90,4 & 79,8 & 65,7 & 76,6 \\
\hline \multirow{3}{*}{$\begin{array}{l}18 \text { a } 24 \\
\text { años }\end{array}$} & 2000 & 26,9 & 25,6 & 40,5 & 31,8 & 14,6 & 28,0 \\
\hline & 2005 & 24,6 & 26,9 & 43,2 & 31,9 & 14,6 & 28,0 \\
\hline & 2013 & 27,6 & 32,1 & 46,8 & 34,4 & 16,6 & 31,9 \\
\hline
\end{tabular}

Fuente: SITEAL 2015 con base en Encuestas de Hogares de cada país. Nota: Los valores correspondientes a NSE para los años cca.2000 y cca.2005 no incluyen a Nicaragua.

\section{COMPROMISOS INSTITUCIONALES CON LA EDUCACIÓN DE CALIDAD COMO DERECHO HUMANO Y VIGILANCIA DE LA SOCIEDAD CIVIL}

Sucesivos pronunciamientos y declaraciones regionales y gubernamentales han impulsado el compromiso con el desarrollo de una educación de calidad para todos (POGGI, 2014: 22). Basten ahora algunos claros ejemplos de ello en esta última década. En la segunda reunión intergubernamental del Proyecto Regional de Educación para América Latina y el Caribe, convocada por la UNESCO en Buenos Aires los días 29 y 30 de marzo de 2007, los ministros y las ministras de Educación de América Latina y el Caribe ratificaban: 
"la necesidad de intensificar los esfuerzos para lograr una educación de calidad para todos, entendida como un bien público y un derecho humano fundamental y una responsabilidad del conjunto de la sociedad." (UNESCO, 2007: 2)

Sobre esa base, reconocen los notables esfuerzos que los países de la región han realizado hasta ese momento:

\footnotetext{
"para eliminar el analfabetismo, aumentar los años de escolaridad obligatoria, ampliar la cobertura en todos los niveles del sistema educativo, mejorar la infraestructura y las condiciones de salud y nutrición de los niños y las niñas, diseñar nuevos currículos, fortalecer la formación profesional de los docentes, y mejorar los sistemas de información, evaluación y administración educativa." (UNESCO, 2007: 2)
}

Sin embargo, forzosamente, han de reconocer que, a pesar esas tareas realizadas, "hay sectores sociales que se encuentran aún en una situación de desigualdad en lo que se refiere al acceso, la prosecución de estudios y los logros de aprendizaje”, una desigualdad que juzgan "incompatible con la construcción de sociedades justas, capaces de incluir a todos, en el marco de procesos de desarrollo económico y social sostenidos y sostenibles". Desde ese punto de vista, consideran que:

\footnotetext{
"el principal desafío de la región es asegurar el derecho de todos a una educación de calidad a lo largo de la vida que sea relevante, pertinente, equitativa y, a través de una acción pública eficaz y eficiente. Este derecho es una opción ética que condiciona nuestro futuro y que ha de basarse en el principio de igualdad de oportunidades y en el derecho a la no discriminación, expresados en la Convención relativa a la lucha contra las discriminaciones en la esfera de la enseñanza." (UNESCO, 2007: 2).
}

Para ello, la mejora de la calidad educación de la educación pública es considerada como fundamental "dado el rol que juega en la reducción de las desigualdades y en el fortalecimiento de la cohesión social". Una educación pública de calidad que ha de ser garantizada por el Estado con, entre otras medidas, un incremento de la inversión educativa, unos sistemas y unos centros educativos más inclusivos, y un compromiso fuerte con la mejora de la formación y situación laboral y profesional de los docentes.

Unos años más tarde, los Ministros y representantes de los Ministerios de Educación de América Latina y el Caribe, reunidos en la Ciudad de México, del 29 al 30 de enero de 2013, para la III Reunión ampliada de la Mesa del Proyecto Regional de Educación para América Latina y el Caribe, plantean un conjunto de recomendaciones para la construcción de la Agenda Educativa Post-2015 en América Latina y el Caribe. Lo hacen en el marco de los logros e incumplimientos tanto de las seis Metas del programa de Educación para Todos como de los Objetivos de Desarrollo del Milenio para 2015 en el terreno de la educación, reconociendo los claros avances pero también los desafíos que persisten por no haber alcanzado las metas y objetivos propuestos. Así, los Ministros reconocen que "la gran 
tarea pendiente en la región es la inequidad y sus consecuencias en el progreso de los países", y asumen que:

"el principio rector que orienta la agenda educativa post-2015 es la reducción de la inequidad y la pobreza por medio de la provisión de una educación de calidad para todos que contribuya a avanzar hacia sociedades más inclusivas.” (UNESCO, 2013: 1)

Partiendo de esas consideraciones, invitan a que la Agenda de la educación post2015, enfatizando que el impacto de la educación se revela como esencial para lograr un desarrollo humano sostenible, impulse una planificación educativa que:

"tome en cuenta las realidades y especificidades nacionales y sub-nacionales, de manera que las estrategias y políticas educativas sean pertinentes y relevantes a los contextos sociales, económicos y culturales de los distintos países, para seguir avanzando en la búsqueda de la equidad social." (UNESCO, 2013: 1)

En la perspectiva del nuevo horizonte post-2015, los Ministros identifican una serie de temas "emergentes" en el terreno de la educación que exigen una adecuada respuesta y desarrollo. De hecho, se constituyen en elementos relevantes de la nueva Agenda educativa regional, entre ellos: una educación para la ciudadanía ampliamente comprehensiva y orientada a los jóvenes; una expansión ampliada de las TICs en los procesos de enseñanzaaprendizaje y en los de gestión de la educación reduciendo al tiempo la brecha digital; desarrollo de programas educativos orientados al aprendizaje a lo largo de la vida; consolidar la expansión de la educación terciaria y superior con programas de calidad adecuadamente relacionados con las actividades de $\mathrm{I}+\mathrm{D}$; desarrollo de programas comprehensivos de educación multicultural e intercultural; y, en seguimiento de RIO+20, generalizar contenidos y prácticas educativas sobre desarrollo sostenible (UNESCO, 2013: 2-3).

Haré referencia ahora, por último, a uno de los más recientes pronunciamientos ministeriales-institucionales en la región. Los Ministros de Educación de América Latina y el Caribe, representantes gubernamentales de alto nivel, agencias de las Naciones Unidas, representantes de organizaciones de la sociedad civil y asociados del desarrollo, que asistieron a la reunión Educación para Todos (EPT) en América Latina y el Caribe: balance y desafíos post-2015, en Lima, Perú, el 30 y 31 de octubre del 2014, terminaron su tarea con la llamada Declaración de Lima. En ella, reiterando previas declaraciones en esa misma línea, vuelve a reafirmarse que:

"la educación es un derecho humano fundamental, la base para la garantía de la realización de otros derechos, y es esencial para la prosperidad y un crecimiento pacífico, inclusivo, equitativo y sostenible en la región." (UNESCO, 2014: 2-4) 
Al mismo tiempo, precisamente por ese valor asignado a la educación, constatando que "el mayor desafío para el progreso social en la región es la desigualdad", consideran que:

"el principio rector de la agenda educativa post-2015 debe ser contribuir a la reducción de la desigualdad y la pobreza a través de la provisión de una educación inclusiva de calidad, y aprendizaje a lo largo de la vida para todos/as, así como también valoramos la riqueza de la diversidad cultural de la región." (UNESCO, 2014: 1)

Junto a ese aprendizaje inclusivo, equitativo y de calidad a lo largo de la vida para todos que proporcione a los jóvenes las habilidades y competencias necesarias para la vida y el trabajo decente, la Declaración reclama también, como en México el año anterior, un impulso especial a la educación para el desarrollo sostenible. Asimismo, se recuerda la importancia de atender las necesidades de formación y mejora del profesorado y de los actores del liderazgo educativo, todos "actores clave para el logro de la calidad de la educación" (UNESCO, 2014: 2-4).

Las organizaciones de la sociedad civil latinoamericana, en especial las agrupadas en torno a la Campaña Latinoamericana por el Derecho a la Educación (CLADE) han jugado un papel muy relevante en la vigilancia de esos compromisos gubernamentales con la garantía del derecho a una educación de calidad como derecho humano irrenunciable, al tiempo que han llevado a las agendas gubernamentales de la región algunos de sus principios y objetivos básicos. Y lo han hecho desde una concepción crítica de la acción educativa que denuncia:

"la concepción del ser humano como capital humano, y de la educación como instrumento al servicio del mercado, con el fin de impulsar la empleabilidad, tendencia que viene consolidándose a nivel mundial." (CLADE, 2012: 2)

Posición plasmada en diversos pronunciamientos a lo largo de los últimos años: Santa Cruz, 2003; Brasilia, 2004; Valparaíso y Panamá, 2007; o Guatemala, 2008. Un buen ejemplo de la esencialidad de sus posiciones se refleja bien en la Declaración de la VII Asamblea de la CLADE, en Quito en abril de 2012, donde reclamaban:

"Una educación pertinente, relevante, transformadora, crítica, debe tener como fin máximo la promoción de la dignidad humana y la justicia social y ambiental. La educación, derecho humano promotor de los demás derechos, debe asumir a niños, niñas, jóvenes y personas adultas como sujetos del derecho, promover la interculturalidad, la igualdad, la equidad de género, el nexo entre ciudadanía y democracia, el cuidado y relación armónica con la naturaleza, la eliminación de toda forma de discriminación, la promoción de la justicia y la construcción de una cultura de paz y de resolución no violenta de los conflictos." (CLADE, 2012: 2) 


\section{LA EDUCACIÓN PARA TODOS EN AMÉRICA LATINA EN 2015}

El último Informe de seguimiento de 2015 del programa de Educación para Todos (EPT) certifica con claridad tanto los avances en educación como sus límites reales en América Latina. Ese diagnóstico para la región pone el foco en dos puntos centrales de la Agenda educativa internacional post-2015 refrendados en la reciente Declaración de Incheon (2015). Tanto la calidad como la equidad, y las estrategias que pueden favorecerlas, deben seguir siendo los objetivos prioritarios en materia de educación en la perspectiva del 2030. El balance sintético que, en 2015, puede hacerse de los avances y estancamientos en la región de los seis objetivos del programa que monitoriza la UNESCO es el que sigue. Con todos los objetivos del programa alcanzados, medidos con el Índice de Desarrollo de Educación para Todos ${ }^{1}$ (IDE) de 2012, sólo se encuentra Cuba (0.981). Ningún país de la región se encuentra lejos de alcanzarlos. Próximos a conseguirlos se encuentran Chile (0.969), Uruguay (0.969), México (0964), la República Bolivariana de Venezuela (0.956), y Ecuador (0.951). En una posición intermedia se sitúan Costa Rica (0.948), Panamá (0.938), el Estado Plurinacional de Bolivia (0.921), Perú (0.913), El Salvador (0.909), Colombia (0.902), Paraguay (0.892), República Dominicana (0.891), Honduras (0.870) y Guatemala (0.850) (UNESCO, 2015a: 11-12).

En relación al primer objetivo, extender y mejorar la atención y educación de la primera infancia, se han producido significativos avances en toda la región, si bien, como es habitual, con notables diferencias nacionales. Globalmente, se ha incrementado la matrícula un 34\%, pasando de los 16 millones en 1999 a los 21 millones de 2012. En ese último año la tasa bruta de matrícula fue de un $74 \%$ frente al $54 \%$ de 1999 . Más de la mitad de los países, entre ellos Chile, Ecuador, México, Cuba, Perú y Uruguay, lograron que la tasa bruta de matrícula en la enseñanza preescolar alcanzara un índice del $80 \%$ o más. Por debajo del $60 \%$ se encuentran Colombia, Honduras y Nicaragua. El nivel más bajo de escolarización en este nivel, por debajo del 40\%, lo encontramos en Paraguay. La presencia del sector privado en este nivel educativo ha descendido en países como Colombia, Ecuador, El Salvador y Guatemala, mientras ha crecido en Perú. Estudios realizados en

\footnotetext{
${ }^{1}$ El índice de desarrollo de la educación para todos (IDE) es "un índice compuesto que proporciona una evaluación global del sistema educativo de un país en relación con los objetivos de la EPT”. En realidad, atendiendo a la disponibilidad limitada de datos, dicho Índice abarca los cuatro objetivos de los seis que pueden cuantificarse con más facilidad. El segundo, la enseñanza primaria universal, medida por la tasa neta de escolarización ajustada en primaria. La primera parte del objetivo cuarto, la alfabetización de los adultos, medida por la tasa de alfabetización de las personas de 15 años y más. El quinto objetivo, la paridad e igualdad entre los sexos, medida por el índice de la EPT relativo al género (IEG), que es el promedio de los índices de paridad entre los sexos de las tasas brutas de escolarización en la enseñanza primaria y secundaria y de la tasa de alfabetización de los adultos. Y, por último, el sexto objetivo, la calidad de la educación, medida por la tasa de supervivencia en el quinto grado de primaria. El valor del IDE para un país determinado, con un máximo de uno, es la media aritmética de los cuatro indicadores que miden cada uno de los componentes ahora señalados (UNESCO, 2011: 294-295). Para 2015, por insuficiencia o carencia de datos no se incluyen en el cálculo del IDE: Brasil, Argentina y Nicaragua.
} 
Argentina, Brasil, Colombia y Perú han mostrado que las intervenciones tempranas en preprimaria muestran efectos muy positivos sobre la probabilidad de una posterior matrícula en primaria, en la evitación de la repetición y abandono escolar — uno de los más graves problemas de los sistemas educativos de la región (CEPAL, 2013: 116) - y en el rendimiento escolar a lo largo de los primeros años de la escuela primaria (UNESCO, 2015a: 2-3).

Probablemente, uno de los más serios problemas que afrontar en los países de la región en esta etapa educativa sea el del bajo nivel de cualificación profesional del personal que trabaja en pre-primaria, en especial en el tramo de edad 3-5 años. La Organización de Estados Americanos (OEA/OAS) señalaba que a pesar de haberse definido estándares para definir la cualificación y competencias para enseñar en este nivel son pocos los países que cuentan con personal adecuadamente preparado (OAS, 2011: 71). Por su parte, la Organización para la Cooperación y el Desarrollo Económico (OCDE) ha considerado que la adecuada cualificación del personal de este nivel educativo es la mejor garantía del derecho de los niños a una educación de calidad (OECD, 2012: 146-148). Con datos de 2010, encontramos que el nivel de cualificación de los profesionales de este nivel varía entre los países de la región. Entre el $90 \%$ y 100\% de maestros cualificados en Colombia, El Salvador y la República Bolivariana de Venezuela. Por encima del $80 \%$ en Costa Rica y México, y más del $70 \%$ en Ecuador. Por el contrario, en Nicaragua y Panamá, menos del $50 \%$ del profesorado que trabaja en ese nivel educativo tiene el nivel de cualificación técnico-profesional adecuado (SIPI, 2014). El problema se torna incluso más agudo para los profesionales que trabajan en el tramo de 0-3 años. Araujo, López-Boo y Puyana analizando 42 programas de atención y educación de la primera infancia dirigidos a niños de 0 a 3 años y sus familias en 19 países de América Latina y el Caribe muestran que el nivel de cualificación adecuado para desempeñar su tarea se da para el $62.5 \%$ de los maestros, para el $30.4 \%$ de Auxiliares del maestro y para el $41.2 \%$ de los cuidadores (ARAUJO, LÓPEZ-BOO and PUYANA, 2013: 55-56).

A propósito del segundo de los objetivos del programa Educación para Todos, lograr la universalización de la enseñanza primaria, especialmente en lo que respecta a las niñas y los niños de ambos sexos pertenecientes a minorías étnicas o marginadas, el último Informe de seguimiento de la UNESCO (2015a: 3-4) establece que más del 50\% de los países de América Latina y el Caribe han logrado la universalización de la enseñanza primaria. No obstante, respecto a 1999 la tasa de matrícula hasta 2012, con 65 millones de niños y niñas matriculados en primaria, ha descendido un $7.5 \%$, aunque la media ajustada de la tasa neta de matrícula se ha mantenido entre el 93\%-94\% en ese periodo. La tasa neta de matrícula en primaria en México, Cuba y Perú está por encima del 97\%; superan el 80\% en Ecuador, El Salvador, Guatemala, Honduras, Nicaragua, Panamá y la República Bolivariana de Venezuela. Por debajo de ese último porcentaje se encuentra Paraguay. Tanto en Colombia como en el Estado Plurinacional de Bolivia se han producido descensos 
significativos en la tasa neta de matrícula desde 1999. La tasa de finalización del último grado de la primaria constituye una preocupante realidad. En 2011 estaba en el 77\%, el mismo valor que en 1999; es decir, la quinta parte de los alumnos de primaria de la región abandonan la escuela antes de haber terminado el ciclo de enseñanza primaria.

En América Latina y el Caribe hay todavía 3,7 millones de niños sin escolarizar en este ciclo de enseñanza; se ha observado una escasa reducción desde los 4 millones de niños desescolarizados en 1999. Aunque en el conjunto de América Latina el número de niños sin escolarizar disminuyó en un 8,9\%, en el Caribe aumentó en un 11,4\%. Merece la pena señalar un dato muy negativo: en 2012 el inacabable conflicto armado que padece Colombia era el responsable del $16 \%$ de no escolarización en toda la región. Como se pone de manifiesto desde la UNESCO, el impacto de los conflictos armados se observa en la proporción de la población educada, la media de años de escolarización y la tasa de alfabetización (UIS/UNESCO, 2010: 4). Para Colombia, los estudios realizados establecen que la población en edad escolar que vive en zonas afectadas por el conflicto armado tiene un año menos de la media de escolarización. En el caso del impacto de las secuelas de la guerra civil en Guatemala los estudios muestran que en términos de educación han sido las mujeres las principales perjudicadas (UIS/UNESCO, 2010: 32 y 34-35). Situación que incluso se ha agravado en los últimos tres años en regiones como el África subsahariana, el Sur y el Oeste de Asia y los países árabes, que, de los 121 millones de niños y adolescentes desescolarizados, aportan 33.8 millones. Globalmente la tasa de desescolarización en primaria en zonas afectadas por conflictos armados ha pasado del 30\% en 1999 al $36 \%$ en 2012 (UNESCO, 2015b: 2).

En el nivel de educación primaria, desde el punto de vista de la reducción de las desigualdades vinculadas a la pobreza-clase social, grupo étnico o residencia se han producido algunos avances. En Guatemala las brechas entre niños indígenas y no indígenas se han acortado entre 2000 y 2011. En Nicaragua la tasa de logro entre los niños de las familias más pobres ha pasado del 16\% en 2001 al 66\% en 2009. El acceso a la escuela primaria para las familias más empobrecidas también ha mejorado en los casos de Brasil, Guatemala, Perú y el Estado Plurinacional de Bolivia, a esto, incluso con limitaciones, han contribuido los Programas de Transferencias Condicionadas.

Acerca del tercero de los objetivos, garantizar que los jóvenes y adultos tengan iguales oportunidades de acceso al aprendizaje y la adquisición de competencias para la vida diaria, considerando el índice de matrícula en el primer ciclo de la enseñanza secundaria, entre 1999 y 2011 la mayoría de los países han incrementado las tasas de transición de primaria a secundaria, países como Ecuador y Panamá lo han hecho en un $25 \%$ o más. Encontramos así que un $13 \%$ de los países de la región han logrado escolarizar a la totalidad de la población en edad de cursar ese ciclo, y otro $17 \%$ se aproxima mucho a este resultado. En conjunto, la tasa bruta de matrícula en el primer ciclo de secundaria ha 
pasado de un 95\% en 1999 a un 98\% en 2012, en países como Costa Rica, Ecuador y Guatemala la tasa en esos años se ha incrementado en más de 30 puntos porcentuales. Esa misma tasa en educación secundaria superior ha pasado del 63\% en 1999 al 76\% en 2012; es aquí destacable el caso de la República Bolivariana de Venezuela que en ese periodo ha incrementado su tasa de escolarización bruta en el nivel del 41\% al 76\% (UNESCO, 2015a: 4).

Bassi, Busso y Muñoz (2013), manejando 113 Encuestas de Hogares en 18 países de América Latina a lo largo de 1990 a 2010, muestran los importantes avances que se han producido en las tasas globales de matrícula en secundaria. No obstante, aunque las tasas de abandono temprano se han reducido - a excepción de Nicaragua y Perú- pasándose de un $41 \%$ a principios de los noventa a un $30 \%$ en 2010, con Ecuador, Brasil y Colombia con las tasas más altas de decrecimiento del abandono escolar temprano, lo cierto es que ese sigue siendo uno de los principales problemas del nivel. Dándose, además, una brecha importante y creciente de género en perjuicio de los chicos que tienen mayores tasas de abandono que las chicas. Agregado a bajas tasas de graduación, baja calidad de la educación ofrecida por comparación con países de otras regiones con similar PIB, e importantes diferencias en el acceso a una educación de calidad según origen social y zona de residencia. Así, las tasas de graduación en secundaria de los jóvenes de las zonas urbanas son un 26\% —en 1999 era un 25\% - más altas que las de los jóvenes que viven en áreas rurales; sólo Chile, Costa Rica, México, Perú y Colombia han reducido algo esa brecha en los últimos años. A pesar de ello, en Perú, por ejemplo, solamente un $43 \%$ de los adolescentes de las zonas rurales termina sus estudios de primer ciclo de secundaria. Por último, esas tasas de graduación, atendiendo al nivel de riqueza de las familias, arrojan un panorama muy desalentador: los jóvenes del quintil de ingresos más altos se gradúan un 33\% más que los jóvenes del quintil de más bajos ingresos. Una brecha que desde 1999 se ha incrementado en siete puntos porcentuales. En Chile, Colombia, México, Nicaragua, y la República Bolivariana de Venezuela sí se han producido algunos avances en la reducción de esa brecha. En el Estado Plurinacional de Bolivia en 2008 el porcentaje de jóvenes de las familias más pobres matriculados en el primer ciclo de enseñanza secundaria era de un $86 \%$, mientras que el de los jóvenes de las familias más ricas llegan al 99\% (BASSI, BUSSO y MUÑOZ, 2013: 3, 10, y 12-13; UNESCO, 2015a: 5). Por su parte, Bentaouet Kattan y Székely (2015), utilizando datos de 18 países de la región, establecían recientemente que mientras la tasa de matrícula en educación secundaria superior se ha incrementado en los últimos años, la proporción de jóvenes que abandonan tempranamente ese nivel de enseñanza, a pesar de unas relativamente favorables condiciones macroeconómicas, ha permanecido persistentemente alta.

Con respecto al cuarto objetivo, lograr en 2015 una reducción del 50\% de los niveles de analfabetismo de la población adulta, la situación no es muy halagüeña. Existen en la actualidad 33 millones de personas adultas que carecen de los conocimientos básicos 
de lectura y escritura. La situación apenas ha cambiado en los últimos años ya que en 2000 eran 38 millones las personas analfabetas. El 55\% de ellas son mujeres, un problema serio en países como Guatemala. A pesar de que los índices de analfabetismo disminuyeron en un $26 \%$ en toda la región, esa cifra se encuentra lejos del 50\% previsto para el logro de este objetivo en 2015. Solamente el Estado Plurinacional de Bolivia y Perú van a alcanzar esa meta establecida en materia de alfabetización en el cuarto objetivo de Educación para Todos. En cambio, países como Colombia y Nicaragua con una reducción de sólo el $26 \%$ distan de alcanzar ese objetivo (UNESCO, 2015a: 5-6).

A propósito del quinto objetivo, suprimir la disparidad entre los sexos y lograr la igualdad entre ellos en la educación, el $60 \%$ de los países de la región ha logrado la paridad entre niñas y niños en la enseñanza primaria, pero en la enseñanza secundaria ese porcentaje es sólo de un 20\%. En la perspectiva de 2015, globalmente, el panorama no es esperanzador. La supervivencia de las chicas en los últimos grados de la primaria es más alta que la de los chicos, en especial en Colombia, la República Dominicana, El Salvador, Honduras, Paraguay y la República Bolivariana de Venezuela. Situación de desventaja que también — sobre todo en los hijos de las familias más pobres - se da en la secundaria haciendo de la región la única del mundo en la que esa brecha es tan acusada. La diferencia en cuanto a los resultados académicos es asimismo bien visible. En Brasil, México y Perú las chicas tienen mejores resultados que los chicos en Lectura. Los chicos, obtienen mejores resultados que las chicas en Matemáticas en Chile, Colombia, México y Perú. En Argentina se da la situación contraria (UNESCO, 2015a: 6-7).

Por último, considerando el sexto objetivo, mejorar la calidad de la educación para todos y obtener resultados de aprendizaje mensurables, el balance de UNESCO destaca el incremento de la participación de los países latinoamericanos en evaluaciones del aprendizaje tanto regionales como internacionales (SERCE/ TERCE, PISA, TIMSS y PIRLS). Al mismo tiempo, en los últimos quince años, el porcentaje de países latinoamericanos y caribeños que efectúan evaluaciones nacionales pasó de un 56\%, entre 1990 y 1999, a un 63\%, entre 2000 y 2013. En otro plano, desde 1999 se ha producido un aumento del $14 \%$ en el número de maestros de primaria de la región, un total de tres millones en 2012. Sin embargo, subsisten problemas en su capacitación profesional y en ese último año la media regional de maestros de primaria profesionalmente capacitados era del $85 \%$.

Desde la perspectiva de la calidad de la educación que ofrecen las escuelas latinoamericanas, se mantienen desigualdades acusadas entre las zonas rurales y las urbanas, por ejemplo, a propósito del grado de aprovechamiento en el aprendizaje de la lectura. Si se comparan los resultados del Segundo Estudio Regional Comparativo y Explicativo (SERCE) de 2006 y los del Tercer Estudio Regional Comparativo y Explicativo (TERCE) de 2013), organizados y coordinados ambos por el Laboratorio 
Latinoamericano de Evaluación de la Calidad de la Educación (LLECE), se observa una tendencia a la reducción de esa desigualdad, por ejemplo, en países como Argentina, Brasil, Costa Rica y Uruguay, mientras en Colombia, Nicaragua y la República Dominicana se mantienen en 2013 las diferencias (UNESCO, 2015a: 7-8).

\section{LA PERSISTENCIA DE LAS DESIGUALDADES EN EDUCACIÓN COMO PROBLEMA Y DESAFÍO REGIONAL}

Sin embargo, los indudables avances en educación que globalmente se han logrado en la región no se han visto acompañados, como he ido consignando previamente, por mejoras generalizadas en la calidad de la educación ni en la disminución de la desigualdad en el acceso y disfrute de la educación de acuerdo al origen social, étnico, la residencia o la pertenencia a minorías. Situación que ha estado y sigue estando claramente condicionada por la desigualdad estructural que marca al conjunto de países de la región, y que, sin duda, constituye hoy por hoy uno de los más importantes problemas y desafíos en educación que afronta la región latinoamericana.

En 2004 un informe del Banco Mundial señalaba a América Latina y el Caribe como una de las regiones del mundo con mayores desigualdades afectando todos y cada uno de los aspectos de la vida diaria de las personas (FERRANTI et al., 2004). Es, en cualquier caso, una desigualdad social y económica con claras raíces históricas (ECLAC, 2010). Ya a principios del siglo XIX Alexander von Humboldt denunciaba la existencia en la región de "una monstruosa desigualdad de derechos y fortunas" (ELLIOT, 1998: 43). Por su parte, Landes, comparando en términos de desarrollo económico los procesos de colonización de América del Norte y de América Latina y el Caribe, determinaba que el proceso había sido claramente favorable al Norte precisamente "por la distribución más equitativa de su riqueza" (LANDES, 1998: 311). Y, a juicio de Pérez Díaz, "América Latina heredó una estructura social desigual y la extremó considerablemente" (2005: 87). Esta desigualdad se expande en un contexto de baja movilidad socioeconómica, y se agudiza favorecida por dinámicas tanto políticas como familiares que no sólo perpetúan el problema sino que lo agravan (UNDP, 2010). Las medidas tomadas para corregir la situación han sido hasta ahora generalmente muy limitadas e ineficaces. De hecho, existe un amplio consenso a propósito de que superar la desigualdad en sus diferentes formas es el mayor desafío estructural para América Latina y el Caribe (ECLAC, 2010; MARTÍNEZRESTREPO y GRAY, 2013). Y es así porque, como señalan los más recientes estudios sobre el tema, la región continúa siendo la más desigual del mundo (GONZÁLEZ y MARTNER, 2012; CEPAL, 2013).

Obvia e inevitablemente, esa desigualdad se proyecta también sobre el terreno educativo. Los grupos y clases más desfavorecidos siguen teniendo resultados académicos más bajos, menos oportunidades de acceso a la educación y están menos tiempo en el 
sistema educativo. Es la realidad que caracteriza la dinámica cotidiana de los sistemas educativos en sus distintos niveles y áreas (RICO, DELAMÓNICA et al., 2010). Con la perspectiva de 2015 y después, la Oficina Regional de Educación de la UNESCO para América Latina y el Caribe (OREALC) señala la existencia de un conjunto de factores que bloquean la expansión y provisión de una educación de calidad para todos. Especialmente, los altos niveles de desigualdad y pobreza junto con el hecho de que existe un alto porcentaje de población que vive en áreas rurales con escaso acceso a servicios como la educación. Además, en todos los países de la región, persisten importantes niveles de desigualdad vinculados al origen social y étnico y a la zona geográfica en la que se habita. En la práctica, los avances para favorecer a los grupos sociales menos favorecidos han sido bastante limitados, y para esos grupos en desventaja, "por comparación con los grupos más favorecidos, su situación no ha mejorado de forma significativa" (OREALC, 2013a: 4).

Bien recientemente, Alicia Bárcena, Secretaria Ejecutiva de la Comisión Económica para América Latina (CEPAL), en el Foro de Rectores de Las Américas 2015, Prosperidad y Educación: El desafío de la Cooperación en las Américas, celebrado en Ciudad de Panamá, 9 y 10 de abril de 2015, denunciaba una vez más el estancamiento en la reducción de la pobreza y la persistencia de altos niveles de desigualdad que, a su juicio, conspiran contra las democracias de la región (BÁRCENA, 2015: 23). En esa misma perspectiva, para la OCDE, la CEPAL y la Corporación Andina de Fomento (CAF) "La educación debe ser percibida no solo como un motor del crecimiento económico, sino también de inclusión social y reducción de la desigualdad”. En ese sentido, consideran que hay que:

\footnotetext{
"Entender la educación como un vector de mayor cohesión social y de crecimiento inclusivo es crítico para América Latina, dado que su trayectoria de desarrollo se verá en gran parte vinculada a las políticas en este campo. En este sentido, una mayor y mejor inversión en educación es una prioridad para los países de la región con el fin de seguir impulsando una mayor cobertura acompañada de mayor calidad." (OCDE, 2014: 16)
}

\section{UN NUEVO PLAZO EN EL COMPROMISO POR UNA EDUCACIÓN DE CALIDAD PARA TODOS: 2030}

Los representantes gubernamentales, no gubernamentales y de la sociedad civil que respaldan la Declaración de Incheon ofrecen al comienzo de ella un buen resumen de la situación mundial en lo que respecta a la situación de los objetivos del programa Educación para Todos y de los Objetivos de Desarrollo del Milenio vinculados a educación: "Reconocemos los esfuerzos realizados, aunque observamos con gran preocupación que estamos lejos de haber alcanzado la educación para todos" (UNESCO/UNICEF/WORLD BANK, 2015c: 1). Reflexión que, como hemos podido comprobar a lo largo de este texto, se ajusta asimismo muy bien al balance latinoamericano en educación de los últimos quince años. Se hace preciso pues redefinir la Agenda mundial en educación en la perspectiva del post-2015. Diecisiete son los Objetivos de Desarrollo Sostenible en el horizonte de 2030 
que serán debatidos y aprobados en el marco de Naciones Unidas en el otoño de 2015. De ellos, el cuarto propone "Garantizar una educación inclusiva, equitativa y de calidad y promover oportunidades de aprendizaje durante toda la vida para todos" y señala sus correspondientes metas. En la Declaración de Incheon se sostiene que la visión que subyace a ese objetivo general:

"se inspira en una concepción humanista de la educación y del desarrollo basada en los derechos humanos y la dignidad, la justicia social, la inclusión, la protección, la diversidad cultural, lingüística y étnica, y la responsabilidad y la rendición de cuentas compartidas." (UNESCO/UNICEF/WORLD BANK, 2015c: 1-2)

Al mismo tiempo hay una clara reafirmación de que:

"la educación es un bien público, un derecho humano fundamental y la base para garantizar la realización de otros derechos. Es esencial para la paz, la tolerancia, la realización humana y el desarrollo sostenible." (UNESCO/UNICEF/WORLD BANK, 2015c: 1-2)

Y un explícito reconocimiento de que:

\begin{abstract}
"la educación es clave para lograr el pleno empleo y la erradicación de la pobreza. Centraremos nuestros esfuerzos en el acceso, la equidad, la inclusión, la calidad y los resultados del aprendizaje, dentro de un enfoque del aprendizaje a lo largo de toda la vida" (UNESCO/UNICEF/WORLD BANK, 2015c, pp. 1-2).
\end{abstract}

Principios que igualmente podemos ver reflejados en el más reciente documento de reflexión de UNESCO, Rethinking Education. Towards a global common good? (MOHAMMED, MORGAN et al., 2015: 37-42).

Son todos fundamentos que han sido asumidos, al menos formalmente, por los distintos gobiernos latinoamericanos y que son impulsados y exigidos desde la sociedad civil. Y sin duda uno de los factores que más claramente compromete y pone en riesgo hacer realidad esos principios compartidos es la ya señalada persistencia de las desigualdades de todo tipo en la región. Margarita Poggi fija un conjunto de mecanismos de segregación social y educativa que contribuyen a la exclusión de niños, niñas, adolescentes y jóvenes: la ausencia de gratuidad en el acceso y la permanencia en la educación obligatoria; las condiciones materiales de las instituciones escolares; los mecanismos de selección (encubierta); las formas de organización de las trayectorias en los sistemas educativos; algunas concepciones de la autonomía escolar; y las prácticas institucionales y pedagógicas en escuelas y aulas (POGGI, 2014: 55-58). El señalamiento de esos mecanismos dibuja, sin duda, un coherente marco de actuación para la definición de las estrategias de intervención multisectoriales necesarias para seguir trabajando en los próximos años en la reducción de las desigualdades educativas en el terreno de la educación formal. En su consideración, la agenda regional en educación se orienta, sobre la base de 
las oportunas y necesarias estrategias políticas de intervención en las desigualdades sociales y educativas, "hacia una búsqueda de la igualdad con atención, en simultaneidad, a las formas de expresión de la diversidad que caracterizan los territorios geográficos y simbólicos en cada uno de los países que la integran" (POGGI, 2014: 75).

En esa perspectiva, sin duda utópica, del compromiso por seguir avanzando en garantizar para todos más tiempo de educación inclusiva de calidad a lo largo de la vida vale la pena recordar la reflexión de Eduardo Galeano:

"La utopía está en el horizonte. Camino dos pasos, ella se aleja dos pasos y el horizonte se corre diez pasos más allá ¿Entonces para qué sirve la utopía? Para eso, sirve para caminar.”

\section{REFERENCIAS BIBLIOGRÁFICAS}

ARAUJO, M.C., LÓPEZ-BOO, F. y PUYANA, J.M. (2013): Overview of Early Childhood Development Services in Latin America and the Caribbean (Washington, D.C.: Inter-American Development Bank).

BÁRCENA, A. (2015): Visión de América Latina y el Caribe: educación para el cambio $\begin{array}{lllll}\text { estructural } & \text { y } & \text { la } & \text { igualdad. } & \text { Extraído de }\end{array}$ (http://www.cepal.org/sites/default/files/presentation/files/150409_presentacion_abi foro_de_rectores_educacion_para_la_igualdad_final.pdf)

BASSI, M., BUSSO, M. y JUAN SEBASTIAN MUÑOZ, J.S. (2013): Is the Glass Half Empty or Half Full? School Enrollment, Graduation and Dropout Rates in Latin America. IDB Working Paper Series No. IDB-WP-462 (Washington, InterAmerican Development Bank). Extraído de http://publications.iadb.org/bitstream/handle/11319/4671/Is\%20the\%20Glass\%20H alf\%20Empty\%20or\%20Half\%20Full\%3f\%20School\%20Enrollment\%2c\%20Grad uation\%2c\%20and\%20Dropout\%20Rates\%20in\%20Latin\%20America.pdf;jsession id=7594E63C2FB173639E56FFD65F184EB0?sequence $=1$

BENTAOUET KATTAN, R. y SZÉKELY, M. (2015): Analyzing the dynamics of school dropout in upper secondary education in Latin America: a cohort approach, Volume 1. Policy Research Working Paper WPS7223 (Washington, World Bank). Extraído de

http://wwwwds.worldbank.org/external/default/WDSContentServer/IW3P/IB/2015/03/26/0902 24b082ca6a45/Rendered/PDF/Analyzing0the00000a0cohort0approach.pdf

CECCHINI, S. (2014): Educación, Programas de Transferencias Condicionadas y protección social en América Latina y el Caribe, en M.C. Feijoó y M. Poggi (coordinadoras), Educación y políticas sociales. Sinergias para la inclusión, pp. 4984. (Buenos Aires, IIPE - UNESCO).

CEPAL (2013): Panorama social de América Latina 2013 (Santiago de Chile, Naciones Unidas). 
CLADE (2012): Carta de Quito. Declaración de la VII Asamblea de la Campaña Latinoamericana por el Derecho a la Educación (CLADE). Extraído de http://campanaderechoeducacion.org/clade2012/wpcontent/uploads/2012/04/Carta de_Quito_FINAL.pdf

ECLAC (2010): Time for Equality: closing gaps, opening trails (Santiago de Chile, United Nations).

ELLIOT, J. (1998): Do the Americas Have a Common History? (Providence, RI, The John Carter Brown Library).

ESPINDOLA, E. y RICO, M.N. (2010): La pobreza infantil un desafío prioritario, Desafíos, 10, pp. 4-9.

FERRANTI, D., PERRY, G.E., FERREIRA, F. y WALTON, M. (2004): Inequality in Latin America and the. Caribbean: Breaking with History? (Washington, D.C., The World Bank).

GONZÁLEZ, I. y MARTNER, R. (2012): “Overcoming the «empty box syndrome». Determinants of income distribution in Latin America", CEPAL Review, 108, pp. 7-26.

LANDES, D.S. (1998): The Wealth and Poverty of Nations. Why some are rich and some so poor (New York, W.W. Norton \& Company).

MARTÍNEZ-RESTREPO, S. y GRAY MOLINA, G. (2013): The High-hanging Fruit of Latin American Progress (New York, UNDP).

MOHAMMED, A.J. y MORGAN, W.J. et al. (2015): Rethinking Education. Towards a global common good? (Paris, UNESCO Publishing).

OAS (2011): Educational panorama 2010: Remaining Challenges (México City, UNESCO, Secretariat of Public Education of Mexico, and the Organisation of American States).

OECD (2012): Starting Strong III-A Quality Toolbox for Early Childhood Education and Care (Paris, OECD Publishing).

OCDE (2014): Perspectivas económicas de América Latina 2015. Educación, competencias e innovación para el desarrollo (París, OCDE/Naciones Unidas/CAF).

OREALC (2013): Situación Educativa de América Latina y el Caribe: Hacia la educación de calidad para todos al 2015 (Santiago de Chile, UNESCO).

OREALC (2013a): Education Agenda Post 2015 Latin America and the Caribbean. January 29-30 (Mexico City, UNESCO, Santiago Office Regional Bureau of Education for Latin America and the Caribbean). Extraído de http://www.unesco.org/new/fileadmin/MULTIMEDIA/HQ/ED/pdf/Ed-agendaPost2015LatinAmericaandtheCaribbean.pdf

PÉREZ DÍAZ, V. (2005): Sueño y razón de América Latina (Madrid, Taurus).

POGGI, M. (2014): La educación en América Latina: logros y desafíos pendientes. Documento básico (Buenos Aires, Santillana). 
RICO, M.N., DELAMÓNICA, E. et al. (2010): Pobreza infantil en América Latina y el Caribe (Santiago de Chile, CEPAL/UNICEF).

SISTEMA DE INFORMACIÓN SOBRE LA PRIMERA INFANCIA EN AMÉRICA LATINA (SIPI) (2014): Extraído de http://www.sipi.siteal.org/indicadores/consulta

SITEAL (2015): 02 Resumen estadístico comentado. Escolarización en América Latina, 2000-2013. Extraído de http://www.siteal.org/sites/default/files/rec_siteal_2_2015_04_28.pdf

UIS/UNESCO (2010): The hidden crisis: Armed conflict and education. The quantitative impact of conflict on education (Montreal, UNESCO Institute for Statistics). Extraído de http://www.uis.unesco.org/Library/Documents/QuantImp.pdf

UNDP (2010): Regional Human Development Report for Latin America and the Caribbean 2010. Acting on the future: Breaking the intergenerational transmission of inequality inequality (San José, Costa Rica, UNDP).

UNESCO (2007): Declaración de Buenos Aires. II Reunión de Ministros del Comité Intergubernamental del Proyecto Regional de Educación para América Latina y el Caribe, PRELAC. Buenos Aires, Argentina 29 y 30 de marzo 2007. ED2007/PRELAC II/REF.1. Extraído de http://unesdoc.unesco.org/images/0015/001599/159986S.pdf

UNESCO (2011): Informe de seguimiento de la EPT en el mundo 2011. Una crisis encubierta: conflictos armados y educación (París, UNESCO). Extraído de http://unesdoc.unesco.org/images/0019/001921/192155S.pdf

UNESCO (2013): Recomendaciones Agenda Educativa Post 2015 en América Latina y el Caribe. México, enero 29-30, 2013. Extraído de http://www.unesco.org/new/fileadmin/MULTIMEDIA/FIELD/Santiago/pdf/recome $\underline{\text { ndaciones-agenda-educativa-post-2015.pdf }}$

UNESCO (2014): Educación para Todos (EPT) en América Latina y el Caribe: Balance y Desafíos post-2015, 30 - 31 de octubre del 2014, Lima, Perú. Declaración de Lima. Extraído

de http://www.unesco.org/new/fileadmin/MULTIMEDIA/FIELD/Quito/pdf/Declaraci on lima.pdf

UNESCO (2015a): EFA Global Monitoring Report. Regional overview: Latin America and the Caribbean (Paris, UNESCO Publishing). Extraído de http://es.unesco.org/gemreport/sites/gem-report/files/LAC_en.PDF

UNESCO (2015b): Education for All Global Monitoring Report. Policy Paper 21. Humanitarian Aid for Education: Why it Matters and Why More is Needed. June 2015 (Paris, UNESCO). Extraído de http://unesdoc.unesco.org/images/0023/002335/233557E.pdf

UNESCO/UNICEF/WORLD BANK (2015c): Declaración de Incheon. Educación 2030: Hacia una educación inclusiva y equitativa de calidad y un aprendizaje a lo largo de 
la vida para todos. ED/WEF2015/MD/3. Extraído de http://unesdoc.unesco.org/images/0023/002331/233137S.pdf

\section{PROFESIOGRAFÍA}

\section{Luis M. Lázaro Lorente}

Catedrático de Teoría e Historia de la Educación. Profesor de la Universidad de Valencia desde 1984. Ha impartido ininterrumpidamente ahí clases de Educación Comparada desde 1986 hasta la actualidad, además de cursos de doctorado y seminarios de Educación Comparada e Internacional en Universidades europeas y latinoamericanas. Ha dirigido proyectos de investigación, Tesis doctorales y publicado libros y artículos sobre educación superior en la OCDE, políticas educativas en países desarrollados y en desarrollo, la educación en Estados Unidos, y problemas de la educación en América Latina. Datos de contacto: Universidad de Valencia. Facultad de Filosofía y Ciencias de la Educación. Dpto. Educación Comparada e Historia de la Educación. Avenida Blasco Ibáñez no 30. (46010) Valencia, España. E-mail: Luis.Lazaro@uv.es; Twt: @luismiguelazaro

Fecha de recepción: 25 de enero de 2016.

Fecha de aceptación: 25 de abril de 2016. 